\title{
Cellular Adequacy for Thyroid Aspirates Prepared by ThinPrep: How Many Cells Are Needed?
}

\author{
Claire W. Michael, M.D., ${ }^{1 *}$ Yijun Pang, M.D., Ph.D., ${ }^{1}$ Robert T. Pu, M.D., Ph.D., ${ }^{1}$ \\ Farnaz Hasteh, M.D., and Kent A. Griffith, M.P.H., M.S. ${ }^{2}$
}

Although it is well established that ThinPrep introduces artifacts to thyroid aspirates, no criteria have been established for adequacy of such specimens. This study evaluates the adequate number of cells needed to establish the correct diagnosis based on ThinPrep alone. A total of 218 thyroid aspirates prepared by $T P$ with surgical pathology follow-up were reviewed. The cellularity was calculated as follows: Count the total number of clusters, randomly select 10 clusters and count each, calculate the average number per cluster and multiply by the total number of clusters. A minimum number of 6 clusters with 10 cells each was arbitrary established to assume adequacy for a definitive diagnosis. Cytologic diagnoses were classified as: Nondiagnostic (ND), cystic contents, thyroiditis, nodular hyperplasia (NH), follicular/Hurthle $(F / H)$ cell lesion, $F / H$ cell neoplasm, and carcinoma: qualify. Histologic diagnoses were classified as: Cyst (colloid or otherwise), thyroiditis, $\mathrm{NH}, \mathrm{F} / \mathrm{H}$ adenoma, $\mathrm{F} / \mathrm{H}$ carcinoma, carcinoma: qualify. Appropriate treatment triage was considered to be clinical for the former 4 cytologic categories and surgical for the latter 3 with ND warranting repeat aspiration. The results were subjected to logistic regressions analysis and contingency tables correlating the number of cells with the cytologic and histologic diagnosis as well as with treatment triage. Cellularity of sample was ranked in 10 deciles according to the number of cells and in 4 quartiles according to the number of clusters. The agreement percentage, for both diagnostic and treatment, was computed for each decile and quartile. 146 (67\%) cases had cells and received a diagnosis while 72 (33\%) were acellular. Of the 146 cases, 21 contained histiocytes or colloid only. 91/146 (62.3\%) were correctly diagnosed and 123/ $146(84.3 \%)$ would have been correctly triaged for treatment based upon the cytologic diagnosis. Samples with 180 cells or fewer had an agreement rate below 50\%. Agreement rate increases to $80 \%$ when cellularity is 180-320. Above 320 agreement rate remains high but not uniformly. Total number of clus-

${ }^{1}$ Department of Pathology, University of Michigan, Ann Arbor, Michigan

${ }^{2}$ Department of Biostatistics, University of Michigan, Ann Arbor, Michigan

*Correspondence to: Claire W. Michael, M.D., Department of Pathology, University of Michigan, Ann Arbor, MI.

E-mail: clairemi@umich.edu

Received 29 August 2007; Accepted 11 October 2007

DOI 10.1002/dc.20768

Published online in Wiley InterScience (www.interscience.wiley.com). ters did not play an independent role and only the number of cells per cluster had a significant correlation with diagnostic agreement. A 25-cell increase in average cells per cluster increases the odds of agreement between diagnoses by $65 \%$. Diagn. Cytopathol. 2007;35:792-797. 2007 Wiley-Liss, Inc.

Key Words: thyroid; fine needle aspiration; adequacy; ThinPrep

ThinPrep is a liquid-based preparation that has been in use by cytopathology laboratories for more than a decade. It is a filtration-based technique that provides a random representative sample on each Prep. It is well documented that ThinPrep introduces cytologic artifacts to thyroid aspirates, among which the most significant are loss of colloid and far less apparent intranuclear pseudo-inclusions. ${ }^{1}$ Also, in manual preparations, it is customary that most of the material is expressed on the conventional smears and the needle rinse is either prepared as cytospins or concentrated into a cell block. In contrast, for aspirates prepared by liquid based methods, the entire sample is rinsed in the collecting media, from which most laboratories prepare one representative Prep and the remaining material is converted into a cell block. To date, there are no documented studies in the literature that scientifically evaluate adequacy criteria for thyroid aspirates prepared by ThinPrep.

The purpose of this study is to evaluate whether a minimum number of cells or clusters could be established as adequate to render a correct cytologic diagnosis for thyroid aspirates prepared by ThinPrep.

\section{Materials and Methods}

\section{Case Selection}

A SNOMED search was performed on the University of Michigan files between the years 1997 and 2001 for thyroid aspirates in which a ThinPrep was performed (with or without conventional smears) and a surgical follow-up was available. A total of 218 cases were identified, and ThinPrep slides were retrieved. All cases were blindly 
Table I. Cytologic Diagnoses

\begin{tabular}{|c|c|c|c|}
\hline Score & Diagnosis & Number of clusters & Other findings \\
\hline 0 & Nondiagnostic & $<6$ & No colloid \\
\hline 1 & Suggestive of colloid nodule & $<6$ & Abundant colloid \\
\hline 2 & Cystic contents & $<6$ & Numerous macrophages \\
\hline 3 & Lymphocytic/Hashimoto's thyroiditis & N/A & Numerous lymphocytes \\
\hline 4 & Nodular hyperplasia & 6 or more & Benign appearing cells, colloid/histiocytes \\
\hline 5 & Follicular/Hurthle cell lesion & 6 or more & Equivocal features and scant colloid \\
\hline 6 & Follicular/Hurthle cell neoplasm & 6 or more & $\begin{array}{l}\text { Loss of honeycombing, microfollicles, } \\
\text { and syncytial sheets }\end{array}$ \\
\hline 7 & Positive for carcinoma, qualify & 6 or more & Features diagnostic of malignancy \\
\hline
\end{tabular}

N/A, not applicable.

reviewed by three reviewers (C.W.M., Y.P., and R.P.), and a new diagnosis strictly based on an established cytologic classification agreed upon by all authors was rendered. The number of cells and clusters were estimated and logged for every case.

\section{Cytologic Diagnoses}

The cytologic diagnosis was solely rendered upon the review of the ThinPreps. A cytologic classification giving a score from 0 to 7 was developed based on the guidelines published by the Papanicolaou Society with minor modifications (Table I). ${ }^{2}$ A minimum of 6 clusters with 10 cells each was arbitrarily established to render a definitive diagnosis of nodular hyperplasia and beyond.

\section{Histologic Diagnoses}

For the purpose of cyto-histologic correlation, the histologic diagnoses were scored from 1 to 7 for statistical analysis (Table II).

\section{Cluster and Cell Counting}

The number of cells was counted based on the method published by Dooley et al. for ductal lavage specimens. ${ }^{3}$ The total number of clusters per Prep was counted. When numerous, the Prep was divided into several sections, one section was meticulously counted, and the number was multiplied by the total number of sections on the Prep. Ten morphologically representative (average sized) clusters were randomly selected and the number of cells in each cluster was counted. The average number of cells for the 10 clusters was calculated and multiplied by the total number of clusters to establish the total number of cells per Prep.

\section{Statistical Analysis}

Agreement between cytologic and histologic diagnosis was modeled using standard logistic regression techniques. Agreement was defined in two ways. First, diagnostic accuracy was defined as an exact one-to-one agreement between the cytologic and histologic diagnoses (Tables I and II). Second, treatment triage was defined such that either clinical or surgical management was cor-
Table II. Histologic Diagnoses

\begin{tabular}{cl}
\hline Score & \multicolumn{1}{c}{ Histologic diagnosis } \\
\hline 1 & Simple cyst of any kind \\
2 & Colloid cyst \\
3 & Lymphocytic/Hashimoto's thyroiditis \\
4 & Nodular hyperplasia (colloid goiter) \\
5 & Follicular/Hurthle cell adenoma \\
6 & Follicular/Hurthle cell carcinoma \\
7 & Other carcinoma, qualify; e.g., papillary, medullary etc. \\
\hline
\end{tabular}

rectly indicated by the cytologic diagnosis based upon the histologic diagnosis at surgery. Diagnoses 1-4 and 5-7 (Table II) were considered appropriate for clinical and surgical follow-up, respectively. For statistical models, the number of cells was categorized based upon deciles of its distributions, resulting in groups with approximately 15 cases. The number of clusters was modeled as quartiles. $P$ values less than or equal to $5 \%$ were considered statistically meaningful.

\section{Results}

Based on the cytologic evaluation, 72 cases were considered nondiagnostic, 21 cases insufficient for a definitive diagnosis, and 125 cases diagnostic. A summary of the cytologic diagnoses is represented in Table III and that of cyto-histologic correlation in Table IV.

\section{Cell Counts}

The epithelial cell count ranged from 5 to 187,368 cells/ Prep. Excluding the 72 nondiagnostic cases, a total of 146 cases were further evaluated for diagnostic accuracy and treatment triage. Ninety-one of the 146 cases $(62.3 \%)$ were correctly diagnosed and of those, 123 cases $(84.3 \%)$ would have received appropriate treatment, i.e., surgical versus clinical follow-up. In order to explore the possible association between the number of epithelial cells in the aspirate sample and a correct cytologic diagnosis (as compared to the histologic diagnosis), categories for the deciles of the distribution were created (Table V). Each decile is composed of approximately 15 cases. The agreement percentage, for both cyto-histologic diagnosis and treatment, was computed for each decile. Clearly, for the lowest two deciles, aspirate samples with 180 or fewer 
MICHAEL ET AL.

Table III. Summary of Cytologic Diagnoses (218 Cases)

\begin{tabular}{llrr}
\hline Adequacy & \multicolumn{1}{c}{ Diagnosis score } & Frequency & Percentage \\
\hline Nondiagnostic $(n=72)$ & 0 (no cells) & 51 & 23.4 \\
& 1 (colloid) & 2 & 0.9 \\
Insufficient for definitive & 2 (macrophages) & 19 & 8.7 \\
diagnosis $(n=21)$ & 1 (colloid) & 15 & 6.9 \\
Sufficient $(n=125)$ & 3 (macrophages) & 6 & 2.8 \\
& 4 (nodular hyperplasia) & 12 & 5.5 \\
& 5 (equivocal) & 69 & 31.7 \\
& 6 (neoplastic) & 15 & 6.9 \\
& 7 (carcinoma) & 4 & 1.8 \\
\hline
\end{tabular}

Table IV. Distribution of the Cytopathologic Aspirate Diagnosis and the Histologic Surgical Diagnosis

\begin{tabular}{|c|c|c|c|c|c|c|c|c|c|c|}
\hline \multirow[b]{2}{*}{ Aspirate diagnosis } & \multicolumn{8}{|c|}{ Surgical diagnosis, $N(\%)$} & \multicolumn{2}{|c|}{ Agreement, $N(\%)$} \\
\hline & 1 & 2 & 3 & 4 & 5 & 6 & 7 & 8 & Diagnostic & Treatment \\
\hline 1 & $2(13.3)$ & 0 & $1(6.7)$ & $9(60.0)$ & $2(13.3)$ & 0 & $1(6.7)$ & 0 & $2(13.3)$ & $2(13.3)$ \\
\hline 2 & 0 & $2(33.3)$ & 0 & $3(50.0)$ & 0 & 0 & $1(6.7)$ & 0 & $2(33.3)$ & $2(33.3)$ \\
\hline 3 & 0 & 0 & $3(25.0)$ & $5(41.7)$ & $3(25.0)$ & 0 & $1(8.3)$ & 0 & $3(25.0)$ & $3(25.0)$ \\
\hline 4 & 0 & $1(1.5)$ & $4(5.8)$ & $49(71.0)$ & $8(11.6)$ & $2(2.9)$ & $5(7.3)$ & 0 & $49(71.0)$ & $49(71.0)$ \\
\hline 5 & 0 & 0 & 0 & $4(26.7)$ & $5(33.3)$ & 0 & $6(40.0)$ & 0 & $9(60.0)$ & $15(100)$ \\
\hline 6 & 0 & 0 & 0 & 0 & 0 & $1(25.0)$ & $3(75.0)$ & 0 & $1(25.0)$ & 4 (100) \\
\hline 7 & 0 & 0 & 0 & 0 & 0 & 0 & $24(96.0)$ & $1(4.0)$ & $25(100)$ & $25(100)$ \\
\hline Overall & & & & & & & & & $91(62.3)$ & $100(68.5)$ \\
\hline
\end{tabular}

Table V. Agreement Between Cytological/Histological Diagnosis and Treatment Follow-Up Based on Cellular Content of Aspirate

\begin{tabular}{rccc}
\hline Decile & Cell range & $\begin{array}{c}\text { Diagnostic } \\
\text { agreement }(\%)\end{array}$ & $\begin{array}{c}\text { Treatment } \\
\text { agreement }(\%)\end{array}$ \\
\hline 1 & $5-96$ & 13.3 & 80 \\
2 & $97-180$ & 40 & 80 \\
3 & $181-320$ & 64.3 & 85.7 \\
4 & $321-462$ & 80 & 86.7 \\
5 & $463-727$ & 78.6 & 85.7 \\
6 & $728-1,214$ & 73.3 & 80 \\
7 & $1,215-1,590$ & 60 & 80 \\
8 & $1,591-2,955$ & 85.7 & 92.9 \\
9 & $2,956-9,362$ & 46.7 & 73.3 \\
10 & $9,363-187,368$ & 85.7 & 100 \\
\hline
\end{tabular}

epithelial cells, the rate of agreement is below $50 \%$. However, for cases with greater than 180 cells, the diagnostic agreement increases, but not uniformly. If we group the first 2 deciles and compare them to the remaining 8 deciles, the diagnostic correlation value is highly significant $(P=0.0001)$ but not with treatment agreement $(P=$ 0.4754). The relationship was not linear, as more epithelial cells did not increase the rate of agreement monotonically. This is illustrated in the seventh and ninth decile groups, which decrease in agreement from the lower decile. This was particularly apparent in the ninth decile, which consisted between 2,955 and 9,362 epithelial cells/ Prep, yet has only a $46.7 \%$ agreement between the cytologic and histologic diagnosis.

The rate of treatment agreement is equal to or is larger than the rate of diagnostic agreement for each decile. Again however, the rate does not monotonically increase with increase in epithelial cell count, although all deciles, 4 to 10 have rates of agreement at or higher than $80 \%$, with the exception of the ninth decile which is again lower with only $73 \%$ agreement in treatment.

Overall, the epithelial cell counts are not significantly associated with the rate of agreement, either diagnostic or treatment-related; statistically, $P$ values are 0.4806 and 0.1151 , respectively. However, there does appear to be a threshold, occurring within the third decile, a cellular range of 181-320, where the rate of agreement increases substantially from a suboptimal rate less than $50 \%$ to a rate at or above $80 \%$. Although agreement is not strictly uniform above 320 cells, the rate of diagnostic and treatment agreement rates are $70.7 \%$ and $84.8 \%$ respectively when compiling the last 8 deciles.

ThinPreps of the ninth decile were re-evaluated to explore the reasons that may have lead to the drop in diagnostic accuracy despite the high cellularity (Table VI). Based on the cytologic finding on these Preps, it appears that some cytologic artifacts well known to ThinPrep were the main contributing factors. In 4 cases diagnosed as thyroiditis, the Preps contained numerous lymphocytes and meutrophils. Upon review of the gross description, these were all bloody aspirates and the lymphocytes may have represented peripheral blood cells that were retained after the blood was hemolysed (Fig. 1). In the 4 papillary carcinomas misdiagnosed by cytology, the cells appeared hypochromatic and relatively well arranged within the fragments. Nuclear grooves were subtle and no intranuclear pseudo-inclusions were noted in any of them (Fig. 
Table VI. The Ninth Decile $(N=15)$

\begin{tabular}{cll}
\hline No. of cases & Cytologic diagnosis & \multicolumn{1}{c}{ Histologic diagnosis } \\
\hline 3 & Nodular hyperplasia & Nodular hyperplasia \\
1 & Thyroiditis & Thyroiditis \\
2 & Papillary carcinoma & Papillary carcinoma \\
4 & Thyroiditis & $\begin{array}{c}\text { Adenoma (2); nodular } \\
\text { hyperplasia (2) }\end{array}$ \\
2 & Nodular hyperplasia & Papillary carcinoma \\
3 & F/H cell lesion & Papillary carcinoma (2); nodular \\
& & hyperplasia (1) \\
\hline
\end{tabular}

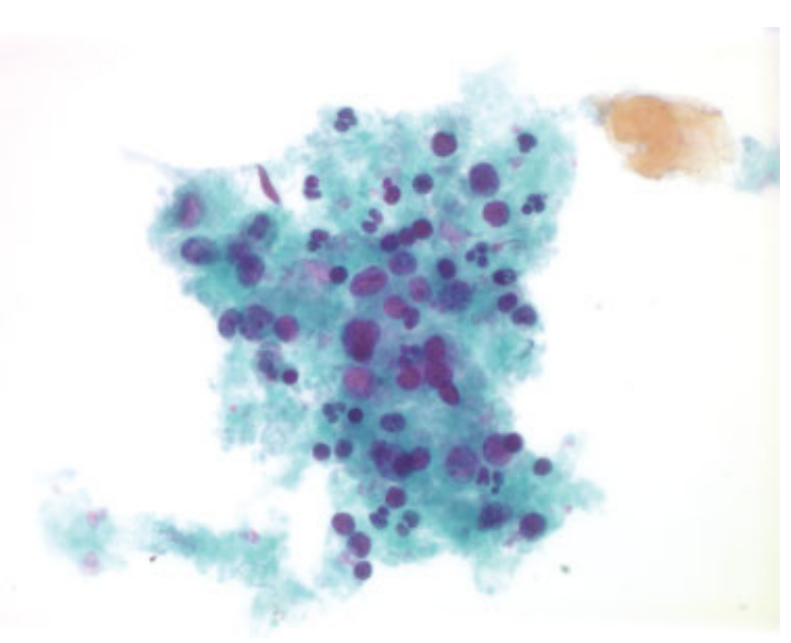

Fig. 1. Follicular/Hurthle cells admixed with mixed inflammatory cells from a case of nodular hyperplasia that was misinterpreted as Hashimoto's thyroiditis. Notice the background of fibrin indicating hemolysed blood. Papanicolaou stain, $\times 600$. [Color figure can be viewed in the online issue, which is available at www.interscience.wiley.com.]

2). In two of those cases, the atypical nature was recognized because of the high cellularity although their papillary nature was missed.

\section{Cluster Count}

The cluster count ranged from 1 to 820 per case. The clusters were distributed into four quartiles based on the number of clusters per case (Table VII) and the number of cells per cluster (Table VIII). By modeling the log odds of an agreement using logistic regression analysis between the aspirate diagnosis and the surgical follow-up diagnosis, using the number of clusters, the average number of cells per cluster, and their interaction as explanatory factors, only the average number of cells per cluster is significantly related $(P=0.0124)$. The model suggests that a 25 cell increase in average cells per cluster increases the odds of agreement between the diagnoses by $65 \%$. The total number of clusters was not significant, and did not appear to play a role in explaining diagnostic agreement (Table IX and Fig. 3). This data suggest that the diagnostic agreement is driven primarily by the number of cells per cluster, rather than the absolute number of clusters.

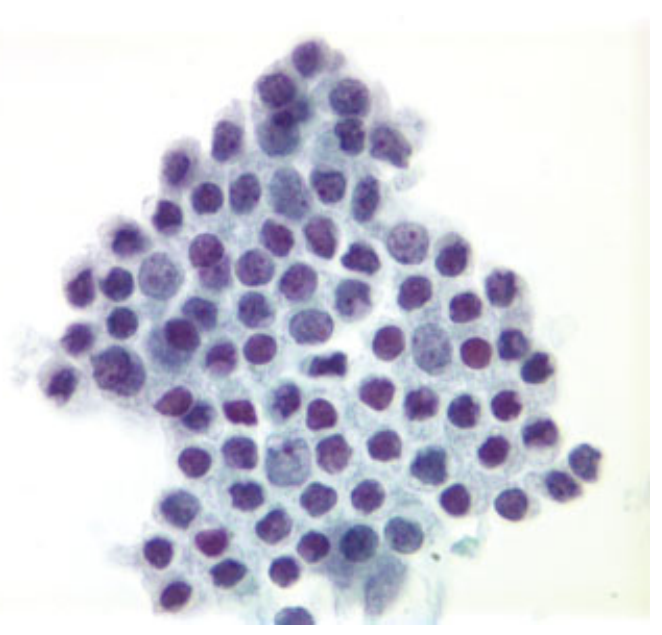

Fig. 2. Cluster of cells with subtle atypia and a honeycomb-like arrangement from a papillary carcinoma misinterpreted as nodular hyperplasia. Notice the nuclear enlargement and membrane irregularity. Papanicolaou stain, $\times 1,000$. [Color figure can be viewed in the online issue, which is available at www.interscience.wiley.com.]

Table VII. Distribution of Clusters $(N=146)$

\begin{tabular}{lcc}
\hline Quartile & Clusters & Cummulative frequency $(\%)$ \\
\hline 1st & $0-8$ & 25.34 \\
2nd & $9-19$ & 50.68 \\
3rd & $20-48$ & 75.34 \\
4th & 49 and above & 100 \\
\hline
\end{tabular}

\section{Discussion}

Thyroid FNA is well established as the first diagnostic procedure in the work-up of a thyroid nodule. ${ }^{4}$ An ultrasound guided FNA is therefore the recommended first step in the work-up of a thyroid nodule with normal or high thyroid stimulating hormone serum levels. With the introduction of easier and more user friendly Ultrasonographic guided aspiration, the number of thyroid FNA have increased dramatically throughout the United States since it can be performed now in both small hospitals and clinics as well as the large facilities. This change of trend has also increased the demand on cytology laboratories to attend these aspirates for adequacy assessment or provide a reasonable way of collection that is practical and yet allow optimal preservation of the sample. The latter option is particularly attractive to megacorporates that transfer specimens across the country to be processed in central laboratories as well as to small laboratories that do not have the proper staffing to afford on site assessment. ${ }^{5}$ Liquid-based preparation technology emerged as a very plausible response to such need. All that is needed is that the clinician collecting the sample rinses the needle in the collecting media and submits it to the laboratory. The collecting media, in our case CytoLyt ${ }^{\mathrm{TM}}$, contains a small concentration of methanol that will allow adequate 
Table VIII. Distribution by Quartile of the Average Number of Cells Per Cluster, by Cluster Quartiles

\begin{tabular}{llll}
\hline Cluster quartile & 25 th & 50 th & 75 th \\
\hline 1st & 19.3 & 33.5 & 51.0 \\
2nd & 22.0 & 34.0 & 63.0 \\
3rd & 24.7 & 38.7 & 51.0 \\
4th & 31.4 & 48.1 & 76.4 \\
All cases & 25.0 & 40.0 & 62.5 \\
\hline
\end{tabular}

Table IX. Agreement Percentage by Cluster Quartile, Stratified by Quartiles for the Average Number of Cells per Cluster

\begin{tabular}{lcccc}
\hline & \multicolumn{4}{c}{ Quartile for the average } \\
\cline { 2 - 5 } Cluster quartile & 1st & 2nd & 3rd & 4th \\
\hline 1st & 28.6 & 58.3 & 60.0 & 33.3 \\
2nd & 33.3 & 70.0 & 88.9 & 36.4 \\
3rd & 50.0 & 80.0 & 76.9 & 100.0 \\
4th & 83.3 & 80.0 & 75.0 & 68.8 \\
\hline
\end{tabular}

preservation of the cells while transferred to the laboratory. The media contains also other hemolytic and mucolytic agents that will allow with further processing a good quality sample with far less obscuring elements than those encountered on conventional smears such as thick blood and inflammation. Also due to the nature of ThinPrep processing, a random representative sample is deposited as a thin layer on the slide. While several studies documented that diagnostic accuracy of FNAs prepared by ThinPrep have improved or at least remained equal to that based on conventional smears, almost all studies acknowledge that ThinPrep introduced cytologic changes to the cellular morphology. ${ }^{6-9}$ These cytologic changes are particularly significant in thyroid FNAs as it impacts the presence and quality of colloid, the presence of intranuclear inclusions, and architectural changes such as breakdown of large papillae into smaller and simpler fragments. These findings however are essential in many of the diagnoses of thyroid nodules by FNA.

In face of these cytomorphologic changes it becomes important that diagnostic ThinPrep features for thyroid FNAs are well characterized by the cytology community. That leads us to a very important question and that is what constitutes an adequate specimen on ThinPrep and is it any different from that of conventional smear? Broadly speaking, an adequate sample should be cellular, representative of the lesion, well preserved and of good quality. In good samples the ThinPrep would provide plenty of cells, random representative Prep, good preservation, and optimum quality without obscuring elements. So the question that remains is how many cells we need to produce an accurate diagnosis and whether a minimum number of cells are required.

The issue of adequacy based on cellularity is controversial based on conventional smears and the cytology community continues to debate on the number of clusters needed to designate an aspirate as adequate. In the guidelines pub-

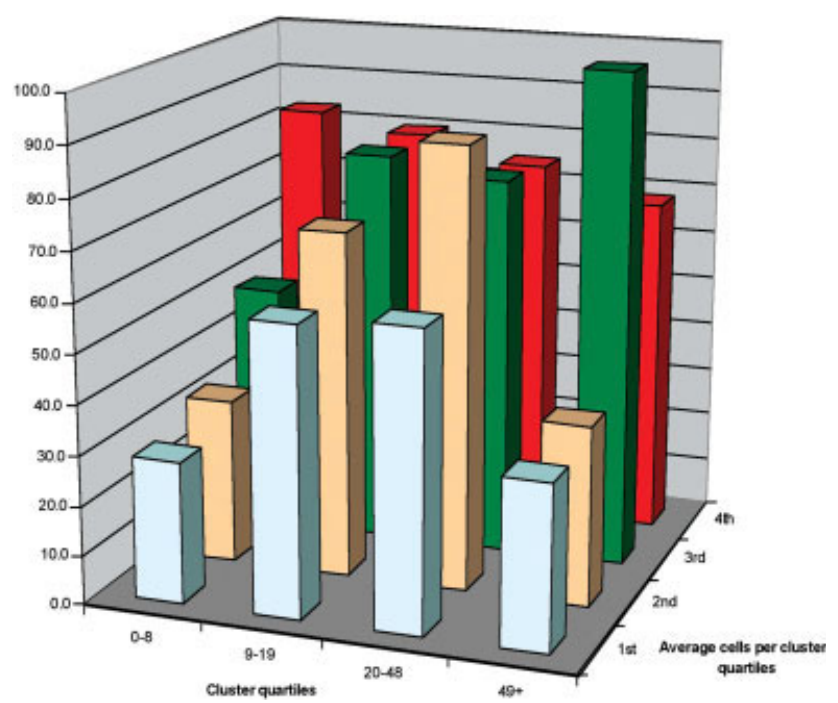

Fig. 3. Agreement percentage by cluster quartiles, stratified by quartiles for the average number of cells. $y$ axis: agreement percentage; $x$ axis: cluster quartiles; $z$ axis: quartiles for the average number of cells per cluster. [Color figure can be viewed in the online issue, which is available at www.interscience.wiley.com.]

lished by the Papanicolaou Society of Cytopathology, the task force acknowledges the fact that criteria for determining adequacy vary from one institution to another and simply refer the reader to criteria recommended in the literature. In summary, these criteria varied from 6 follicular clusters each consisting of 10 cells, ${ }^{10}$ to 10 clusters consisting of 20 cells each, ${ }^{11}$ to a minimum of $6-10$ clusters on two separate smears. ${ }^{12}$ It remains however a fact that most studies document that false diagnosis could be avoided if more stringent adequacy criteria are followed. ${ }^{13}$ Our study was designed to statistically evaluate the validity of specifying a minimum number of clusters or cells on the ThinPrep as adequacy criterion and if so how many is needed.

Based on our study, the epithelial cell count is not significantly associated either with the diagnostic or treatment agreement. However, there is a threshold at the cell range of 180-320, where agreement increases substantially. In fact, when the two first deciles were grouped and compared with the remaining 8 deciles, the diagnostic agreement was highly significant. Based on this finding we have instituted in our institution a minimum requirement of 200 cells to designate a thyroid ThinPrep as adequate.

Most of the literature defines adequacy by a minimum number of clusters, and some go as far as indicating a minimum number of cells per cluster. Goellner et al. ${ }^{10}$ require 6-8 well preserved follicular clusters with at least 10 cells each while Sidawy et al. define adequacy as the presence of 10 clusters with 20 cells each on two separate slides or more. ${ }^{14}$ Kini recommends based on her extensive experience a minimum of 8-10 fragments on two separate slides. ${ }^{15}$ These criteria would translate to a range between 
100 and over 1,000 cells. Interestingly, our study found that the number of clusters did not play an independent role in explaining diagnostic agreement. However, the model suggested that a 25 cell increase in average cells per cluster increased the odds of diagnostic agreement by $65 \%$. Keep in mind that this increase is in the odds of agreement, defined as the probability of agreement divided by the probability of disagreement. For example, if the probability of agreement was $50 \%$ for cases with an average of 25 cells per cluster, the odds of agreement are 1 at this level. With an increase of 25 cells to an average cell count per cluster of 50 , the models suggests the odds of agreement increases by $65 \%$, suggesting a probability of agreement of approximately $62 \%$. This is not cytologically surprising since larger fragments will reveal more about the architectural features of the lesion than small ones. These fragments would also allow better evaluation of the cellular arrangement within the group that is essential in distinguishing hyperplastic from neoplastic lesions, e.g., honeycomb versus syncytium and features such as nuclear molding and overlap.

Surprising however was the dramatic drop of diagnostic correlation in hypercellular samples, particularly the ninth decile where correlation dropped from $85.7 \%$ to $46.7 \%$. Upon second review of these cases, it became clear that while very cellular, the cytologic features necessary for the correct diagnosis were not clear. In particular, papillary carcinoma was noted to present as sheets that appeared honeycomb-like, the nuclei were hypochromatic, bland and the nuclear inclusions were not noted. Upon closer examination however, the fragments while apparently orderly arranged are relatively denser with nuclei compared to what would be expected in a true honey comb. Although nuclear grooves in these cases were not easily seen, the nuclei appeared shrunken with irregular nuclear membranes. In summary, the presence of densely populated sheets should prompt the reviewer to a more meticulous evaluation of the nuclear features at higher magnification. This emphasizes the need to develop the diagnostic criteria as well as the experience in evaluating these specimens.

Another pitfall was lymphocytic thyroiditis, where the presence of increased number of lymphocytes remaining from the hemolysed peripheral blood could be mistaken in negative aspirates as evidence of thyroiditis. Conversely, lymphocytes from thyroiditis tend to be filtered and their number is dramatically diminished when compared to correlating conventional smears.

The presence of false diagnoses despite of the high cellularity is not new. Sidawy et al. ${ }^{14}$ reported 25/133 aspirates with discrepant diagnosis; of these 11 cases met the adequacy criteria yet were described as suboptimal due to other obscuring factors such as blood or thickness of smear. The remaining errors were related to misinterpretation or overlapping criteria. They stress that the simple designation of number of clusters is misleading as other factors inherent to the lesion could interfere with the correct diagnosis. We certainly agree with the latter statement.

In conclusion, a correct diagnosis is not made by number of cells alone, but by the presence of numerous features that can be put together. Yet it seems that at least 200 cells are needed to achieve good diagnostic correlation and that the larger the fragments the better the correlation.

\section{References}

1. Michael CW, Hunter B. Interpretation of fine-needle aspirates processed by the ThinPrep technique: Cytologic artifacts and diagnostic pitfalls. Diagn Cytopathol 1999;23:6-13.

2. Papanicolaou Society of Cytopathology Task Force on Standards of Practice. Guidelines of the Papanicolaou Society of Cytopathology for the examination of fine-needle aspiration specimens from thyroid nodules. Diagn Cytopathol 1996;15:84-89.

3. Dooley WC, Ljung B-M, Veronesi U, et al. Ductal lavage for detection of cellular atypia in women at high risk for breast cancer. J Natl Cancer Inst 2001;93:1624-1632.

4. Cooper DS, Doherty GM, Haugen B, et al. Management guidelines for patients with thyroid nodules and differentiated thyroid cancer. The American Thyroid Association Guidelines. Thyroid 2006; 16(2):109-142.

5. Nasuti JF. Utility of the ThinPrep technique in thyroid fine needle aspiration: Optimal vs. practical approaches. Acta Cytol 2006;50:3-4.

6. Biscotti CV, Hollow JA, Toddy SM, Easley KA. ThinPrep versus conventional smear cytologic preparations in the analysis of thyroid fineneedle aspiration specimens. Am J of Clin Pathol 1995;104:150-153.

7. Cochand-Priollet B, Prat J-J, Polivka M, et al. Thyroid fine needle aspiration: The morphological features on ThinPrep slide preparations. Eighty cases with histologic control. Cytopathol 2003;14:343-349.

8. Tulecke MA, Wang HH. ThinPrep for cytologic evaluation of follicular thyroid lesions: Correlation with histologic findings. Diagn Cytopathol 2004;30:7-13.

9. Malle D, Valeri R-M, Pawaitou-Panajiotou K, Kiziridou A, Vainas I, Destouni C. Use of a thin-layer technique in thyroid fine needle aspiration. Acta Cytol 2006;50:23-27.

10. Goellner JR, Gharib H, Grant CS, Johnson DA. Fine needle aspiration cytology of the thyroid. Acta Cytol 1987;31:587-590.

11. Nguyen GK, Ginsberg J, Crockford PM. Fine-needle aspiration biopsy cytology of the thyroid. Its value and limitations in the diagnosis and management of solitary thyroid nodules. Pathol Ann 1991;26:63-91.

12. Hamburger JI, Husain M. Semiquantitative criteria for fine-needle biopsy diagnosis: Reduced false-negative diagnosis. Diagn Cytpathol 1988;4:14-17.

13. Caraway NP, Sneige N, Samaan NA. Diagnostic pitfalls in thyroid fine-needle aspiration: A review of 394 cases. Diagn Cytopathol 1993;9;345-350.

14. Sidawy MK, Del Vecchio DM, Knoll SM. Fine-needle aspiration of thyroid nodules. Correlation between cytology and histology and evaluation of discrepant cases. Cancer (Cancer Cytopathol) 1997;81:253-259.

15. Kini SR. Guides to clinical aspiration biopsy: thyroid, 2nd edition. New York: Igaku-Shoin; 1996. 